\title{
Time varied illumination laser speckle contrast imaging
}

\author{
Máté Siket ${ }^{1}$, Imre JánokI ${ }^{1}$, Kornél Demeter ${ }^{2}$, Miklós Szabó ${ }^{3}$, and Péter Földesy ${ }^{1, *}$ \\ ${ }^{1}$ Institute for Computer Science and Control, Kende utca 13-17, H-1111, Budapest, Hungary \\ ${ }^{2}$ Institute of Experimental Medicine, Szigony utca 43, H-1083, Budapest, Hungary \\ ${ }^{3}$ First Department of Paediatrics, Semmelweis University, Bókay János u. 53-54, H-1083, Budapest, Hungary \\ *Corresponding author: foldesy.peter@sztaki.hu
}

Compiled December 15, 2021

\begin{abstract}
Laser Speckle Contrast Imaging is a technique to determine blood flow rate with a limitation of low dynamic range. In this Letter, we introduce a varied illumination speckle contrast imaging method. It utilizes varying illumination during exposure to customize the correlation time (flow rate) to speckle contrast relation. The method can cover an order of magnitude larger range flow rate in a single exposure compared to constant illumination methods. The proposed method enables high dynamic range flow rate imaging that is advantageous in studying larger vessels and small arteries. We demonstrate the theory by simulations, ex vivo and in vivo measurements. (๑) 2021 Optical Society of America
\end{abstract}

OCIS codes: (110.0110) Imaging systems; (030.6140) Speckle; (110.6150) Speckle imaging.

http://dx.doi.org/10.1364/ao.XX.XXXXXX

\section{INTRODUCTION}

Laser Speckle Contrast Imaging (LSCI) is an optical technique that has been widely used for monitoring disease induced blood flow changes on the brain surface [1, 2], skin [3], surgery situation [4] or even retina [5]. It has a large field of view, paired with high spatial and temporal resolution, and it requires no contrast agent, which makes it an ideal choice for flowmetry. LSCI is based on the dynamic light scattering of coherent light illumination. Laser speckles can be observed when an illuminated medium scatteres the light in a way that it produces a random interference pattern from the superposition of phase fluctuations. As the pattern changes in accordance with the movement of the scatterers, the decorrelation of such patterns can be used to determine the flow rate of blood. The first description of Laser Speckle Imaging and blood flowmetry was published as early as 1981 [6]. Ever since the technique has been improved greatly. Temporal analysis can help to reduce the time-invariant noise and static scattering [1]. Multi-exposure methods (MESI) $[7,8]$ were developed to increase the dynamic range of the measurements and for static scattering elimination. MESI and its variants use multiple exposure times with constant light intensity to estimate the correlation time of speckles algorithmically and thus the flow rate. To suppress measurement noise and remove static scattering with the aim of obtaining true flow speckle contrast and flow speed, LSCI system calibrations and calculation procedures were proposed $[9,10]$. In order to balance the temporal and spatial resolution, and speckle versus pixel size ratio various solutions have been published [11, 12]. Recently, in [13], a high-speed camera enabled imaging method (DLSI) was presented for wide-field measurement of the speckle temporal intensity autocorrelation function.

For the quantification of speckle contrast, usually the variance of intensity is normalized by the mean intensity in local spatial or temporal windows. This contrast is than used to calculate the speckle autocorrelation (correlation time). The common methodology is the following:

$$
\kappa=\frac{\sigma_{s}}{\langle I\rangle}
$$

where $\kappa$ is the calculated contrast from the image, $\sigma$ is the standard deviation and $\langle I\rangle$ is the mean light intensity over a window of pixels on the image or sequence of images. Relative flow can be then estimated using Eq. (1), as the decorrelation increases with the flow rate and hence $\kappa$ is larger at smaller velocities and vice versa. The normalized second order model of the underlying physical model was presented in [14] as:

$$
v_{2}(T)=\frac{\beta}{T^{2}} \int_{0}^{T} \int_{0}^{T} \rho\left|g_{1}^{n}\left(t^{\prime}-t^{\prime \prime}\right)\right|^{2}+(1-\rho)\left|g_{1}^{n}\left(t^{\prime}-t^{\prime \prime}\right)\right| d t^{\prime} d t^{\prime \prime},
$$

where $v_{2}$ is the reduced second moment, $T$ is the exposure time, $\rho$ is the fluctuating component fraction and $(1-\rho)$ is the statically scattered ratio, and $t^{\prime}-t^{\prime \prime}$ give the time differences during the exposure. The constant $\beta$ is a normalization factor depending on the speckle size, detector size and additional factors of the experimental setup. $g_{1}$ is the electric field autocorrelation [6] which according to [13] gives us $g_{1}^{n}(t)=\exp \left(-\left(t / \tau_{c}\right)^{n}\right)$, where $\tau_{c}$ is the correlation time. The value of $n$ depends on the type of flow dynamics. In case of small arteries/veins in the size range of $\approx 30$ to $150 \mu \mathrm{m}$ with single scattering unordered motion (SU) or multiple scattering ordered motion regime (MO) $n=1$. For multiple scattering unordered motion (MU) representing the behavior of capillary perfused tissue (i.e. parenchyma) $n=0.5$, while $n=2$ describes single scattering from ordered motion for larger vessels (SO). In transition situations, these dynamics $\left(g_{1}^{0.5}, g_{1}^{1}, g_{1}^{2}\right)$ are weighted accordingly extending Eq. (2). The speckle contrast Eq. (1) can be used to identify $\tau_{c}$ and other parameters by modeling $\kappa\left(T, \tau_{c}\right)$ and $\sqrt{v_{2}\left(T, \tau_{c}\right)}$ equivalency. 


\section{THEORY OF TIME VARIED ILLUMINATION SPECKLE CONTRAST IMAGING MODEL}

We investigate the idea of varying laser illumination during a single exposure. We incorporate the varying light intensity into the model of the normalized second order moment, further generalizing it. Furthermore, we substitute the continuously varying light intensity with binary (on/off) level pulse modulation showing its advantages and limitations as well. In practice, binary modulation enables wider intensity range to be covered with higher precision and avoid the burden of calibrating the nonlinear characteristics of analog modulation.

Our scope of work does not include calibration and denoising, as our method could be further inserted into such processes as [10] to achieve quantitative results. Based on the findings, we collectively call the general technique as Time Varied Illumination Speckle Constrast Imaging (TVI-LSCI).

\section{A. Intensity modulation}

The standard LSCI methods have the assumption that the light intensity does not change throughout the exposure time. Note, this holds for the MESI [7] technique, its derivatives, and for single-shot acquisition MESI with a high frame rate singlephoton avalanche diode camera speckle imaging [8] as well. In these methods, constant average laser intensity is used within an exposure.

In order to model the varying laser intensity during a single exposure, we generalize Eq. (2) by defining $I(t)$ as time dependent illumination intensity:

$$
v_{2}(T)=\frac{\beta}{\int_{0}^{T} \int_{0}^{T} I\left(t^{\prime}\right) I\left(t^{\prime \prime}\right) d t^{\prime} d t^{\prime \prime}} \int_{0}^{T} \int_{0}^{T} I\left(t^{\prime}\right) I\left(t^{\prime \prime}\right) g^{\rho, n}\left(t^{\prime}-t^{\prime \prime}\right) d t^{\prime} d t^{\prime \prime},
$$

where $I\left(t^{\prime}\right)$ and $I\left(t^{\prime \prime}\right)$ are the laser intensities at the sample at time instant $t^{\prime}$ and $t^{\prime \prime}$, and for better readability the complex relation including scattering and flow dynamics is substituted as $g^{\rho, n}\left(t^{\prime}-t^{\prime \prime}\right)=\rho\left|g_{1}^{n}\left(t^{\prime}-t^{\prime \prime}\right)\right|^{2}+(1-\rho)\left|g_{1}^{n}\left(t^{\prime}-t^{\prime \prime}\right)\right|$ for nontransition dynamics [13]. The description of the equation intuitively is the following: the decorrelation is weighted with the intensity levels, which can be transformed to be dependent on the time lag by calculating the correlation function. By applying integration once in Eq. (3) the reduced second moment becomes:

$$
v_{2}(T)=\frac{\beta}{\int_{0}^{T} R_{I I}(t) d t} \int_{0}^{T} R_{I I}(t) g^{\rho, n}(t) d t,
$$

where $R_{I I}(t)$ is the autocorrelation of $I(t)$. Note, that selecting $I(t)=$ const. the $R_{I I}(t)$ and the denominator integral reduce to the well known $(1-t / T)$ and $2 / T$ formulas. This equation can be used to derive various contrast to speckle correlation time relation in an efficient way.

We propose as the most advantageous usage of changing illumination profile to be a decreasing valued function. Meaning, a short bright period with decreasing brightness afterwards continuously or in discrete steps. The idea behind is similar to MESI concept, maintaining the average brightness for increasing exposure times. Thus, various $\tau_{c}$ periods are captured with $\propto 1 / \tau_{c}$ intensity and $\propto \tau_{c}$ observation time. The significant difference from the multiple exposures is that the speckle decorrelation phenomena is continuously observed and is not separated into time segments.

\section{B. Pulse modulation}

Our proposed method - similar to the standard speckle contrast imaging - gives valid result under the assumption that, except for the varying intensity, the critical laser properties are maintained such as coherence length, wavelength, noise performance during exposure. In lieu of the limited and usually expensive analog modulation schemes (e.g. laser current modulation, micro-opto-electro-mechanical systems or micromirror array, Bragg cell), we rely on binary intensity modulation of finite pulse time resolution and constant intensity.

The pulse coding enables wider average intensity range to be covered, and at the same time the calibration of the nonlinear characteristics of continuous modulation can be avoided. Given high enough pulse rate and sharp pulse skew, various modulation techniques such as pulse width, position, code or delta modulation can be applied to tailor the speckle contrast and correlation time relation. The validity of the nanosecond pulsed speckle contrast imaging with uniform pulse rate has already been proven in [15].

The pulsed illumination comes with limitations as well. The correlation time may be close to the finite pulse width resulting in distortion in temporal averaging and in $\beta$ [13]. We estimate this limit, derived from the frame-rate requirements of [13], as $\tau_{c} / T>4$, where the $\beta$ becomes near distortion-free of this effect. Thus, e.g. $T=5 \mu$ s pulses enable to capture $\tau_{c}>20 \mu$ s dynamics.

\section{Numerical simulations}

A simulation was done with exposure time of $2 \mathrm{~ms}$ at constant laser intensity (continuous wave, $\mathrm{CW}$ ) and a single exposure with the decreasing illumination value $(I \propto 1 / t)$ using Eq. (4). The $v_{2}$ and $\tau_{c}$ relations are presented in Fig. 1 . In the simulations, three different realistic flow dynamics were considered: $S O_{n=2}, S U / M O_{n=1}$ regimes with $\beta=0.3,1-\rho=0$ values, and $M U_{n=0.5}$ with $\beta=0.3,1-\rho=0.2$ [13]. Fig. 1b) shows the absolute sensitivity $S_{a}$, defined as $v_{2}$ sensitivity to absolute flow changes [16], at the different physiological regions and dynamics.

The sensitivity of the $\mathrm{CW}$ illumination remains localized in a $\tau_{c}$ region raising the need for changing the exposure time to capture rather different flow speeds. The varied illumination on the other hand flattens the sensitivity curves and shifts the sensitive parts of the different dynamics into $\tau_{c}$ regions where they actually occur: $S O_{n=2}$ curve towards lower $\tau_{c}$ of larger flow rate, etc. As a result, we expect that our method compresses and enhance the flow speed coverage while having sensitivity at various dynamics in the physiologically important span [17].

\section{EXPERIMENTAL SETUP}

Two experiments were conducted: first, human full blood was pumped through a clear channel slide, and second, the blood flow in the vessels visualized on a surgically prepared mouse brain surface. The samples were illuminated with a fast modulated continuous laser diode and observed with a camera.

In details, the laser spot was generated by a non-collimated single-mode laser diode (RLD84PZJ2, $\lambda=842 \mathrm{~nm}, 220 \mathrm{~mW}$, ROHM Co., Ltd., Japan). The laser diode was placed in a mount with thermoelectric cooling stage (HLD001, Thorlabs, Newton, NJ, USA). The images were acquired with 10x and $4 \mathrm{x}$ infinity corrected objectives (Thorlabs, Newton, NJ, USA) and a monochrome camera of $3.45 \times 3.45 \mu \mathrm{m}^{2}$ pixel size (Basler ACA2040-55um, Basler Vision Technologies, Germany). The minimal laser pulse length was $T_{o n}=5 \mu s$ (200 KHz sampling 

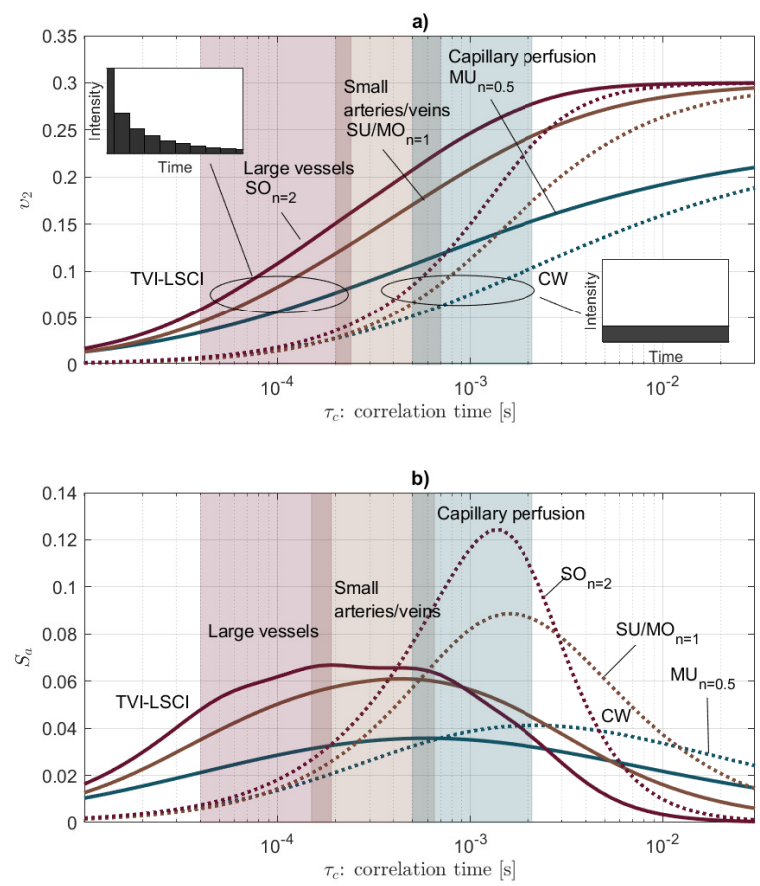

Fig. 1. Simulation of $2 \mathrm{~ms}$ constant illumination (dotted curves) and single-shot $2 \mathrm{~ms}$ time varied intensity illumination (solid curves) speckle contrast with $\beta=0.3,1-\rho=0.2$ for $n=0.5$ and $\beta=0.3,1-\rho=0$ for $n=1,2$ regimes. Fig. a) shows the physiological regions, dynamics types, and the speckle contrast versus correlation time relations. Fig. b) shows the absolute sensitivity of the different dynamics versus illumination scheme.

rate). Interfacing between a host computer, the camera trigger and the pulse waveform generation were done by a data acquisition card (USB-6211, National Instruments Co., Austin, TX, USA). The timing and stability performance of the setup was verified by a Si fixed gain detector (PDA015A/M, Thorlabs, Newton, NJ, USA) and a spectrometer (AvaSpec-ULS2048 StarLine, Avantes Ltd., The Netherlands).

\section{A. Ex vivo experiment}

In the first experiment, a syringe pump (SN-50F6, Sino MedicalDevice Technology Co., Ltd., China) drove the blood through a $\mu$-Slide I Luer channel slide (ibidi GmbH, Germany). The measured medium was room-temperature anticoagulated (citrate) packed red blood cell product for transfusions. The flow velocity was varied across a large dynamic range, from 0 to $600 \mathrm{~mL} / \mathrm{min}$ in 19 logarithmic distributed steps (considering the flow control resolution of the syringe pump). These volumetric flow rates resulted in 0 to $37.5 \mathrm{~mm} / \mathrm{s}$ velocities, taking into account the dimensions of the channel. The visible area was $720 \times 540 \mu \mathrm{m}^{2}$ and the speckle contrast was then calculated over a large region of interest of uniform speed distribution using Eq. (1) [18].

\section{B. In vivo experiment}

In the in vivo experiment, first we deeply anesthetized a mouse (strain: C57BL6/J) by $25 \mathrm{mg} / \mathrm{kg}$ xylazine and $125 \mathrm{mg} / \mathrm{kg} \mathrm{ke}-$ tamine in $0.9 \% \mathrm{NaCl}$ mixture, and then we fixed the head of the animal in a stereotaxic frame (Stoelting Inc, USA). Next we made a $1 \mathrm{~cm}$ long sagittal incision on the skull, and cleaned the surface by $3 \% \mathrm{H}_{2} \mathrm{O}_{2}$. With a high speed drill we opened the skull and made a $5 \times 5 \mathrm{~mm}$ window on the parietal part. To avoid the drying of the tissue, we put physiological saline solution on the brain and covered it with a glass cover slip. The used $4 x$ objective resulted in appr. $4 \times 3 \mathrm{~mm}$ useful visible area on the brain surface. The speckle size was $s \approx 13.2 \mu \mathrm{m}$.

\section{RESULTS}

\section{A. Model Identification and Pulse Modulation Optimization}

First, the model given in Eq. (4) has been identified based on controlled measurements of the ex vivo setup. In the second step, to make the effect of an intensity profile predictable, we used the identified model for optimization and used it later on the in vivo measurement.

We defined $\tau_{c}:=k \frac{1}{v+v_{b}}$, where $v$ is the flow speed in the channel, $v_{b}$ is the speed corresponding to Brownian motion and $k$ is a scaling factor, $\rho=1$ neglecting the static scattering component. As the exploration of second type dynamics induced variability is not in the scope of this article, we assumed $n=1$ for the ex vivo characterization. For the identification Eq. (4) was extended with a $v_{S}$ offset as proposed in [7], to better account for static scatterers and measurement noise. The identification process was formulated as an optimization problem, where the free variables are: $\beta, k, v_{s}$ and $v_{b}$. The goal of the optimization was to minimize the root-mean-square error (RMSE) between the measured and the simulated contrast values:

$$
\min _{\beta, k, v_{b}, v_{s}} \sum_{i=1}^{M} R M S E\left(\boldsymbol{\kappa}^{(i)}-\boldsymbol{\kappa}_{\text {sim }}^{(i)}\right),
$$

where $M$ is the number of different exposure times, $\kappa$ is the measured and $\kappa_{\text {sim }}$ is the simulated contrast. The optimization was done by the MATLAB 2020a fmincon function interior-point method from 20 initial points generated by Latin Hypercube Sampling (LHS) [19]. The identified parameters were given as $\beta=0.195, k=6.027 \cdot 10^{-4} \mathrm{~mm}$ and $v_{b}=0.0072 \mathrm{~mm} / \mathrm{s}$ with RMSE of $0.012 \pm 0.0047$.

During intensity profile optimization the goal was to extend the dynamic range of the non-saturating speckle contrast values from the $\tau_{c} \approx 10^{-5}-10^{-2} \mathrm{~s}$ span. A given exposure time has been sliced into sections of different lengths, and each section could have different repetition rate of the fixed length pulses (i.e. equivalent CW constant intensity). From an optimization point of view the variables were the length and duty cycle of each section. A feasible implementation requires the lengths to be integer multiplicative of the hardware limited pulse width $\left(T_{o n}=5 \mu \mathrm{s}\right)$, thus the number of pulses in a particular section becomes $N_{i}=\operatorname{round}\left(\frac{T_{i} D_{i}}{T_{o n}}\right)$, where $T_{i}$ is the section length and $D_{i}$ is the duty cycle. These pulses are then equally distributed on the resulting $T_{i}^{h}=\operatorname{round}\left(\frac{N_{i} T_{o n}}{1-D_{i}}\right)$, where subscript $h$ indicates the hardware implemented section length. The rounding introduces discontinuities in the cost function rendering derivative based optimizer not applicable. Therefore, instead of the fmincon algorithm, patternsearch was applied from 20 initial points generated by the LHS, similarly to the identification process. The cost function and optimization was defined in the following way: 


$$
\begin{aligned}
& \min _{T_{i}, D_{i}} \sigma\left(\frac{\Delta \kappa\left(T, \tau_{c}\right)}{\Delta t_{c}}\right)+\mu\left(\frac{\Delta \kappa\left(T, \tau_{c}\right)}{\Delta t_{c}}\right)^{-1}, \\
& \text { subject to } t_{c}=\log \left(\tau_{c}\right), \\
& \sum_{i} T_{i} \cdot D_{i}=T_{\text {const }},
\end{aligned}
$$

where $T_{\text {const }}$ is the total "on time" of the equivalent exposure using one constant frequency, $\sigma(\cdot)$ is the standard deviation and $\mu(\cdot)$ is the mean. The first term of the cost function Eq. (6) forces the convergence of the difference quotients toward a constant value, realizing a linear-like response on the logarithmic scale. The second term forces the speckle contrasts to spread, by maximizing the mean slope of the response. The Eq. (8) puts a constraint on the exposure time. A change of variable is made $\left(t_{c}\right)$, since the near-linear response is expected on the logarithmic scale, where the differences are calculated between the experimentally evaluated flow speeds. The simulated and measured speckle variances are illustrated in Fig. 2 labeled with the different illumination scenarios. The curves represent a single exposure per rate with no averaging of multiple frames. Due to the lack of continuous illumination control in the setup, the constant illumination cases in the comparison were produced by uniformly distributed pulse trains similarly to [15].

It can be observed that the resulting correlation time span has higher dynamic range compared to the uniformly distributed illumination setups as expected.

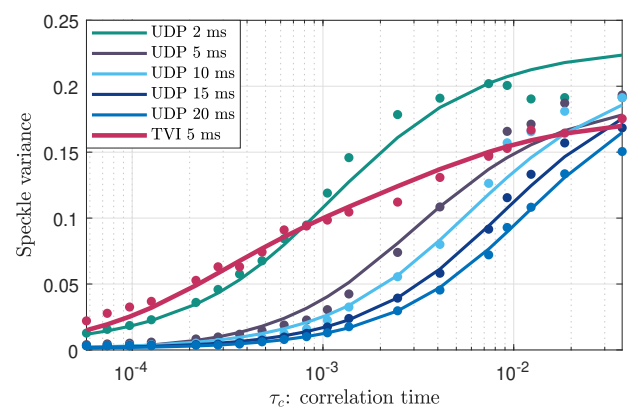

Fig. 2. Measured (dots) and simulated (lines) speckle variances given different exposure times of uniformly distributed pulse trains (UDP) and time varied illumination (TVI) profile as a function of flow rate.

\section{B. In Vivo Experiment Results}

The protocol was the following during the experiment: after the preparation of the animal, the optical setup is adjusted and a sequence of 100 frames was captured for each measurement scenarios and then the stored sequences were evaluated. The scenarios contained continuous and modulated pulse train illumination versions as well. Before capturing a series, we adjusted the laser position in order to use the broadest dynamic range of the camera at the given exposure time with no saturating pixel values. Finally, the speckle contrast was then calculated with Eq. (1) on a $7 x 7$ pixels sliding windows in the individual frames and to improve the signal-to-noise ratio we averaged across the contrast maps.

The results are presented in Fig. 3. The images show that for such relatively small flow speed ranges present in the prepared mouse cranial window our TVI-LSCI method perform similarly to CW LSCI. However, it is expected based on our simulations and ex vivo experiment, that in scenarios with higher flow speed ranges like in the human circulatory system TVILSCI can achieve better speckle variance and gaining advantage by its higher dynamics mapping. Such experiments will be carried and evaluated in the future.
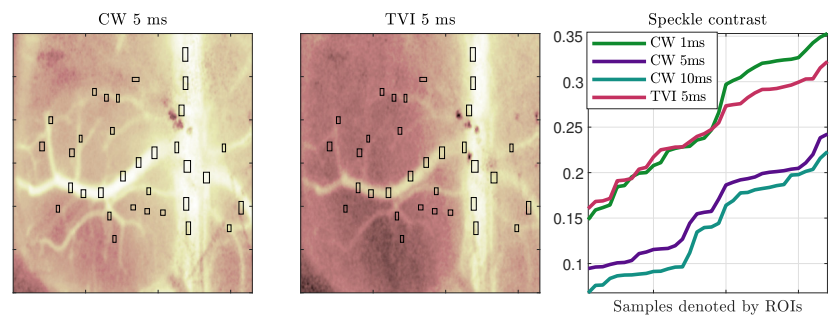

Fig. 3. In vivo experiment showing speckle contrast of the surface of a mouse brain. Image captions indicate continuous illumination (CW) and varied illumination (TVI-LSCI) outcomes at different exposure time and pulse train length. The ROIs are registered on each image and picked at different vessel size positions. The shown field of view is $3.2 \times 3.2 \mathrm{~mm}^{2}$.

\section{DISCLOSURES}

The authors declare no conflicts of interest.

\section{REFERENCES}

1. P. Li, S. Ni, L. Zhang, S. Zeng, and Q. Luo, Opt. Lett. 31, 1824 (2006).

2. M. A. Taylor, T. Nöbauer, A. Pernia-Andrade, F. Schlumm, and A. Vaziri, Optica 5, 345 (2018).

3. B. Choi, N. M. Kang, and J. Nelson, Microvasc. Res. 68, 143 (2004).

4. C. Zheng, L. W. Lau, and J. Cha, Biomed. optics express 9, 5962 (2018).

5. H. Cheng and T. Q. Duong, Opt. Lett. 32, 2188 (2007).

6. A. Fercher and J. Briers, Opt. Commun. 37, 326 (1981).

7. A. B. Parthasarathy, W. J. Tom, A. Gopal, X. Zhang, and A. K. Dunn, Opt. Express 16, 1975 (2008).

8. T. Dragojević, D. Bronzi, H. M. Varma, C. P. Valdes, C. Castellvi, F. Villa, A. Tosi, C. Justicia, F. Zappa, and T. Durduran, Biomed. Opt. Express 6, 2865 (2015).

9. P. Zakharov, A. Völker, A. Buck, B. Weber, and F. Scheffold, Opt. Lett. 31, 3465 (2006).

10. C. Wang, Z. Cao, X. Jin, W. Lin, Y. Zheng, B. Zeng, and M. Xu, Biomed. Opt. Express 10, 4097 (2019).

11. O. Thompson, M. Andrews, and E. Hirst, Biomed. optics express 2 , 1021 (2011)

12. H. Cheng, Q. Luo, S. Zeng, S. Chen, J. Cen, and H. Gong, J. biomedical optics 8, 559 (2003).

13. D. D. Postnov, J. Tang, S. E. Erdener, K. Kılıç, and D. A. Boas, Sci. Adv. 6, eabc4628 (2020).

14. R. Bandyopadhyay, A. S. Gittings, S. S. Suh, P. K. Dixon, and D. J. Durian, Rev. Sci. Instruments 76 (2005).

15. Y. Zhao, K. Wang, W. Li, H. Zhang, Z. Qian, and Y. Liu, J. Biomed. Opt. 25, 1 (2020).

16. S. Yuan, A. Devor, D. A. Boas, and A. K. Dunn, Appl. optics 44, 1823 (2005).

17. D. A. Boas and A. K. Dunn, J. Biomed. Opt. 15, 011109 (2010).

18. J. W. Goodman, "Statistical properties of laser speckle patterns," in Laser speckle and related phenomena, (Springer, 1975), pp. 9-75.

19. R. L. Iman, "Latin hypercube sampling," in Wiley StatsRef: Statistics Reference Online, (American Cancer Society, 2014). 


\section{FULL REFERENCES}

1. P. Li, S. Ni, L. Zhang, S. Zeng, and Q. Luo, "Imaging cerebral blood flow through the intact rat skull with temporal laser speckle imaging," Opt. Lett. 31, 1824-1826 (2006).

2. M. A. Taylor, T. Nöbauer, A. Pernia-Andrade, F. Schlumm, and A. Vaziri, "Brain-wide 3d light-field imaging of neuronal activity with speckleenhanced resolution," Optica 5, 345-353 (2018).

3. B. Choi, N. M. Kang, and J. Nelson, "Laser speckle imaging for monitoring blood flow dynamics in the in vivo rodent dorsal skin fold model," Microvasc. Res. 68, 143-146 (2004).

4. C. Zheng, L. W. Lau, and J. Cha, "Dual-display laparoscopic laser speckle contrast imaging for real-time surgical assistance," Biomed. optics express 9, 5962-5981 (2018).

5. H. Cheng and T. Q. Duong, "Simplified laser-speckle-imaging analysis method and its application to retinal blood flow imaging," Opt. Lett. 32, 2188-2190 (2007).

6. A. Fercher and J. Briers, "Flow visualization by means of singleexposure speckle photography," Opt. Commun. 37, 326-330 (1981).

7. A. B. Parthasarathy, W. J. Tom, A. Gopal, X. Zhang, and A. K. Dunn, "Robust flow measurement with multi-exposure speckle imaging," Opt. Express 16, 1975-1989 (2008).

8. T. Dragojević, D. Bronzi, H. M. Varma, C. P. Valdes, C. Castellvi, F. Villa, A. Tosi, C. Justicia, F. Zappa, and T. Durduran, "High-speed multiexposure laser speckle contrast imaging with a single-photon counting camera," Biomed. Opt. Express 6, 2865-2876 (2015).

9. P. Zakharov, A. Völker, A. Buck, B. Weber, and F. Scheffold, "Quantitative modeling of laser speckle imaging," Opt. Lett. 31, 3465-3467 (2006).

10. C. Wang, Z. Cao, X. Jin, W. Lin, Y. Zheng, B. Zeng, and M. Xu, "Robust quantitative single-exposure laser speckle imaging with true flow speckle contrast in the temporal and spatial domains," Biomed. Opt. Express 10, 4097-4114 (2019).

11. O. Thompson, M. Andrews, and E. Hirst, "Correction for spatial averaging in laser speckle contrast analysis," Biomed. optics express 2, 1021-1029 (2011).

12. H. Cheng, Q. Luo, S. Zeng, S. Chen, J. Cen, and H. Gong, "Modified laser speckle imaging method with improved spatial resolution," J. biomedical optics 8, 559-565 (2003).

13. D. D. Postnov, J. Tang, S. E. Erdener, K. Kılıç, and D. A. Boas, "Dynamic light scattering imaging," Sci. Adv. 6, eabc4628 (2020).

14. R. Bandyopadhyay, A. S. Gittings, S. S. Suh, P. K. Dixon, and D. J. Durian, "Speckle-visibility spectroscopy: A tool to study time-varying dynamics," Rev. Sci. Instruments 76 (2005).

15. Y. Zhao, K. Wang, W. Li, H. Zhang, Z. Qian, and Y. Liu, "Laser speckle contrast imaging system using nanosecond pulse laser source," J. Biomed. Opt. 25, 1-10 (2020).

16. S. Yuan, A. Devor, D. A. Boas, and A. K. Dunn, "Determination of optimal exposure time for imaging of blood flow changes with laser speckle contrast imaging," Appl. optics 44, 1823-1830 (2005).

17. D. A. Boas and A. K. Dunn, "Laser speckle contrast imaging in biomedical optics," J. Biomed. Opt. 15, 011109 (2010).

18. J. W. Goodman, "Statistical properties of laser speckle patterns," in Laser speckle and related phenomena, (Springer, 1975), pp. 9-75.

19. R. L. Iman, "Latin hypercube sampling," in Wiley StatsRef: Statistics Reference Online, (American Cancer Society, 2014). 